\title{
Energy-aware routing for biomedical wireless sensor networks
}

\author{
Carlos Abreu ${ }^{\mathrm{a}, *}$, Manuel Ricardo ${ }^{\mathrm{b}}$, P.M. Mendes ${ }^{\mathrm{c}}$ \\ a Escola Superior de Tecnologia e Gestão, Instituto Politécnico de Viana do Castelo, Portugal \\ ${ }^{\mathrm{b}}$ INESC TEC, Faculdade de Engenharia, Universidade do Porto, Portugal \\ c Centro Algoritmi, Universidade do Minho, Portugal
}

\section{A R T I C L E I N F O}

\section{Article history:}

Received 15 February 2013

Received in revised form

26 August 2013

Accepted 23 September 2013

Available online 17 October 2013

\section{Keywords:}

Energy-aware routing

Network lifetime

\section{Quality of service}

Routing for low-power and lossy networks

Biomedical wireless sensor networks

\begin{abstract}
A B S T R A C T
Available wireless sensor networks targeting the domain of healthcare enables the development of new applications and services in the context of E-Health. Such networks play an important role in several scenarios of patient monitoring, particularly those where data collection is vital for diagnosis and/or research purposes. However, despite emerging solutions, wearable sensors still depend on users' acceptance. One proposed solution to improve wearability relies on the use of smaller sensing nodes, requiring more energy-efficient networks, due to smaller room available for energy sources. In such context, smaller wireless sensor network nodes are required to work long time periods without human intervention and, at the same time, to provide appropriate levels of reliability and quality of service. Satisfaction of these two goals depends on several key factors, such as the routing protocol, the network topology, and energy efficiency. This paper offers a solution to increase the network lifetime based on a new Energy-Aware Objective Function used to design a Routing Protocol for Low-Power and Lossy Networks. The proposed Objective Function uses the Expected Transmission Count Metric and the Remaining Energy on each sensor node to compute the best paths to route data packets across the network. When compared with state of the art solutions, the proposed method increases the network lifetime by $21 \%$ and reduces the peaks of energy consumption by $12 \%$. In this way, wireless sensor network nodes wearability can be improved, making them smaller and lighter, while maintaining the required performance.
\end{abstract}

(c) 2013 Elsevier Ltd. All rights reserved.

\section{Introduction}

A Wireless Sensor Network (WSN) is a distributed, selforganised network of small, energy-constrained, wireless nodes that interact to carry out complex tasks (Akyildiz et al., 2007). A Biomedical Wireless Sensor Network (BWSN) is a small-size WSN equipped with biomedical sensors designed for medical applications or healthcare services (Abreu et al., 2012). Typical applications of BWSNs include patient monitoring, catastrophe and emergency response, as well as Ambient Assisted Living (AAL) for disabled or elderly people (Hadjidj et al., 2013; Lai et al., 2009).

A BWSN can be seen as a physical layer for Healthcare Information Systems (HISs), collecting data to support healthcare professionals on their decisions and medical diagnosis. In this context, BWSN integration with the existing network infrastructure is essential to provide ubiquity and pervasiveness to the HIS (Preve, 2011). The integration of BWSNs with traditional networks based on the Internet Protocol (IP) was made possible thanks to the contributions of the working group IPv6 over Low Power Wireless Personal Area Networks (6LoWPANs), created by the Internet

\footnotetext{
*Corresponding author. Tel.: +351 258819 700; fax: +351258 827636 .

E-mail address: cabreu@estg.ipvc.pt (C. Abreu).
}

Engineering Task Force (IETF). It was the main objective of 6LoWPAN to define an adaptation layer that should make possible the integration of the IP version 6 (IPv6) over low power WSNs based on the IEEE 802.15.4 technology (Hui and Thubert, 2011). The use of 6LoWPAN BWSNs integrated with HIS to implement patient monitoring applications and services is now a possibility, as portrayed in Fig. 1. It is important to notice that, unlike Body Sensor Networks (BSNs), BWSNs have no data concentrator (e.g., smartphone or wearable computer) on each patient. BWSN sensor nodes autonomously route the data packets through the network and deliver them at a central or local database.

The miniaturisation of the BWSN nodes and sensors along with the absence of a data concentrator improves the wearability of the monitoring system. However, it is necessary to develop a strategy to extend the network lifetime. An extended period of BWSN work without human intervention (e.g., battery changing) is desirable because frequent interventions on multiple nodes are likely to hamper users' acceptance. Furthermore, longer network lifetime will decrease BWSN operational costs.

WSNs are different from traditional wireless networks in several aspects. Their nodes have low computational and energy resources, the communication channels have narrow bandwidths, and the wireless links may be exposed to high levels of interference. These intrinsic characteristics bring new challenges to the 


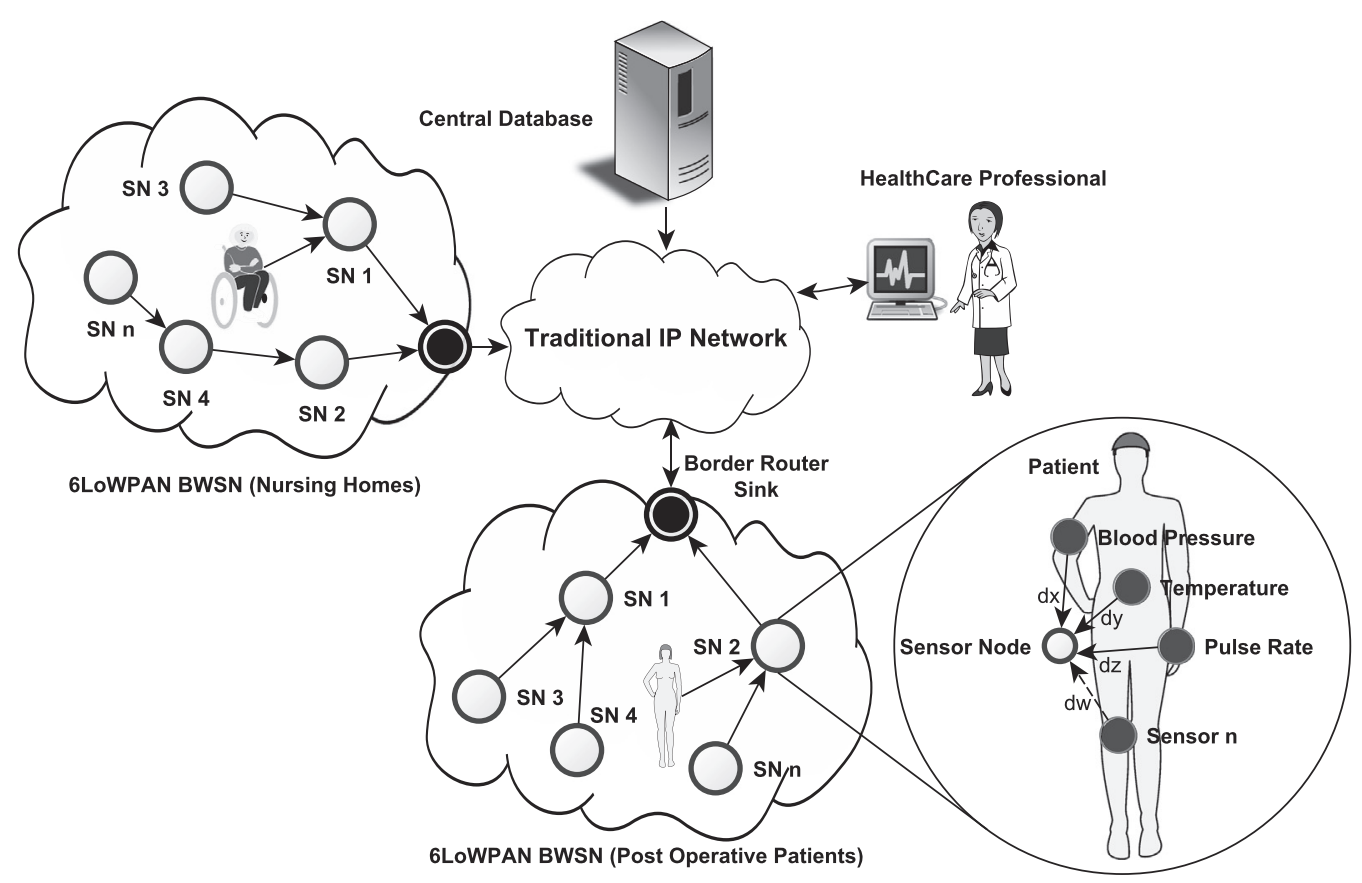

Fig. 1. Integrating 6LoWPAN BWSNs into the healthcare information system.

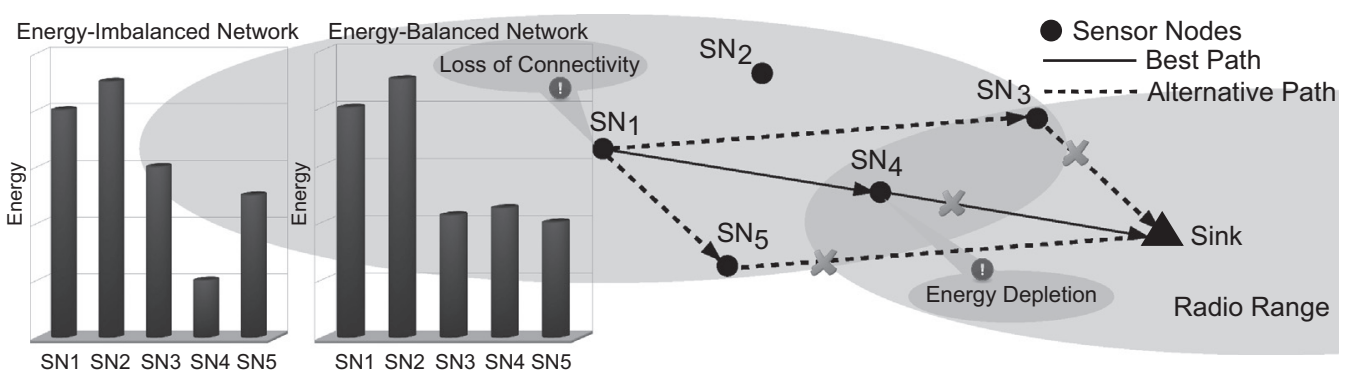

Fig. 2. Remaining energy distribution in imbalanced and balanced networks.

design of WSN including their routing protocol (Watteyne et al., 2011). The routing protocols are responsible for the selection of paths through the network; they also contribute to determine the communications reliability and efficiency, and to energy efficiency.

The research and industrial community has proposed several routing protocols for WSNs. The most recent is the Routing Protocol for Low-Power and Lossy Networks (RPL), proposed by the IETF working group Routing Over Low-power and Lossy Networks (ROLL) (Winter and Thubert, 2012). The RPL is a versatile Distance Vector IPv6 routing protocol that uses an Objective Function (OF) to specify how network nodes select their parents and, consequently, select paths to route the packets through the network (Winter and Thubert, 2012). The versatility of RPL allows different OFs being used according to both the network application and the application requirements.

Since WSNs should operate on very scarce energy resources, most of the proposed routing protocols focus on finding energyefficient paths so as to increase the network lifetime (Bicakci and Tavli, 2010; Ok et al., 2010). Since energy efficiency may not guarantee increased lifetime to the network, in order to avoid the overuse of the most efficient paths and balance the energy consumption on the network it is necessary to consider the remaining energy on each sensor node (Ok et al., 2009, 2010). Considering a WSN with five Sensor Nodes (SNs) and one Sink (Fig. 2), when $S N_{1}$ sends its messages to the sink, it may attempt to send it only through $\mathrm{SN}_{4}$ because $\mathrm{SN}_{4}$ is its best parent and belongs to the most energy-efficient path to the Sink, what may lead $S_{4}$ to inactivity due to energy exhaustion (Abdulla et al., 2012). Nevertheless, an energy-balanced network where alternative paths are used will allow the network to remain active and fully functional for a longer period of time, since all sensor nodes remain active.

In order to achieve energy efficiency and balance, we propose a new Energy-Aware OF (EAOF) for the RPL routing protocol. To the best of our knowledge, this is the first implementation and evaluation of an energy-aware OF to the RPL routing protocol over 6LoWPAN BWSNs. Regarding a patient monitoring scenario, the proposed EAOF prolongs the network lifetime and reduces the need for human intervention on battery changing, thereby enhancing the system wearability and reducing operational costs.

In what follows, the motivation behind this work is presented (Section 2), and then the routing challenges and design issues in BWSNs are outlined (Section 3). In Section 4, the RPL protocol is briefly presented, and then the EAOF is presented and evaluated (Section 5). Finally, the implementation results are analysed and some conclusions are drawn.

\section{Motivation}

Patients monitoring in general or step-down hospital units as well as in nursing rooms or other health care facilities is crucial to avoid clinical worsening of inpatients. In most cases, vital or physiological signs (e.g., temperature, pulse and respiratory rate, blood pressure and oximetry) are measured manually by nursing 
professionals a few times a day. This time consuming task can be complemented in an efficient and reliable way by using real-time monitoring systems, as depicted in Fig. 1.

As stated, the gathering of vital or physiological signs can be done by using BWSNs. To make this an everyday reality, BWSN sensor nodes have to fulfil a demanding set of requirements, being the sensor nodes wearability one of the most important. The aspects related with nodes wearability are crucial to improve users' acceptance of the technology, and the size of the sensor nodes is key in that regard. They must be as small as possible, but unfortunately, the use of small sensor nodes may have a strong drawback. In fact, since the energy module tends to use a substantial part of the overall volume of the sensor node, and energy is one of the scarcest resources in WSNs, its use may result in a reduction of the network lifetime.

Communications are one of the most energy consuming tasks in WSNs. Consequently, it is crucial to use adequate data paths from the sensor nodes to the sink in order to improve the network lifetime. In recent years, many different approaches have been used to design energy-efficient routing protocols, e.g., clusterbased methods (Liu et al., 2012; Ferng et al., 2012), data aggregation (Lindsey and Raghavendra, 2002), energy-efficient paths (Shah and Rabaey, 2002) and geographic information-based algorithms (Xu et al., 2001). More recently, the scientific community has been proposing new protocols that achieve energy efficiency as well as energy balance among the sensor nodes in the network (Ok et al., 2009, 2010; Ren et al., 2011; Tao et al., 2012). These studies agree that energy-aware routing algorithms must be distributed, self-organised, reliable, energy-efficient, and improve the energy balance in the network, bearing in mind that multi-hop communications are at stake here. Moreover, to achieve such goals, especially those related to energy efficiency and balance, the energy-aware routing protocols must explicitly take into account the node's remaining energy.

The standard for IPv6 routing in low-power WSNs, i.e. the RPL, computes the best paths to route the data through the network according to an objective function and a set of metrics. These metrics can be either node attributes, such as hop-count, node remaining energy; or link attributes, such as link quality, latency, and expected transmission count (ETX) (Vasseur and Kim, 2012). Among these metrics, the ETX is widely used to design reliable routing protocols for WSNs since it reflects the quality of the paths used to transmit the data. The hop-count and the ETX are the only metrics used to implement the objective functions related to the RPL, i.e. the Objective Function Zero (Thubert, 2012) and the Minimum Rank Objective Function with Hysteresis (Gnawali and Levis, 2012). None of these objective functions use an energy based metric to compute how to form paths to route the data through the network.

Considering the aforementioned requirements and limitations, this paper proposes an Energy-Aware Objective Function for the RPL. As already discussed, the path ETX and remaining energy are strictly necessary to design reliable and energy-efficient routing protocols. Thus, the proposed objective function uses both the remaining energy on each node and the path ETX as metrics to achieve energy efficiency and balance in a distributed, self-organised and reliable way, while choosing the most reliable links to route the data through the network, in order to increase the network lifetime.

\section{Routing challenges and design issues in biomedical wireless sensor networks}

While important technologies being considered, WSNs and their applications still present challenges to the industrial and research community because of their intrinsic characteristics, such as low energy resources, limited bandwidth, unstable wireless links, low computational power and small memory. These characteristics present a major obstacle to the development of reliable, easy-to-implement routing protocols (Watteyne et al., 2011; Ko et al., 2011c). As a special set of WSNs, BWSNs share the abovementioned challenges and they add some others, depending on their purpose and application.

\subsection{Wireless link}

Harsh environments like hospital facilities are prone to radiofrequency interference and shadows due to the presence of medical equipment, obstacles and human bodies. Such adverse conditions make the Signal to Noise plus Interference Ratio (SNIR) experienced by the network nodes low and unstable. Combined with the low modulations-coding adaptability of the sensor network technologies, this fact leads to links having high and variable Frame Error Ratio (FER). In turn, the instability of wireless links leads to path instability, which also contributes to an increase of the Packet Error Ratio (PER) that makes communications unreliable or prone to large delays, depending on the retransmission policy in use. The routing protocol, which is the component in charge of selecting network paths, must address this instability in order to maintain communication paths reliable without overreacting to temporary link instability.

\subsection{Network deployment and topology}

Network deployment in BWSNs depends on their applications or on the scenario. In its turn, the network topology may be called deterministic or random. Deterministic deployments are used in AAL and patient monitoring applications; in these cases, the sensor nodes are manually placed in locations that have a minimum level of radio coverage and link quality (to this end, some connectivity tests should be performed in advance). Random deployments are used in disaster or emergency response scenarios where the sensor nodes are required to create an ad hoc, possibly multi-hop network. In both situations, and since from a medical viewpoint it is indifferent whether the former or the latter deployment scenario is on the ground, the network must guarantee adequate levels of reliability and Quality of Service (QoS).

\subsection{Nodes mobility}

Patients under monitoring may have some degree of mobility. Mobility could result in link quality degradation, isolation of the sensor node, or topology changes. The network should be able to cope with some degrees of mobility so that sensor nodes are not isolated and communications can be provided with satisfactory levels of QoS.

\subsection{Network lifetime}

BWSNs are typically composed of dozens of battery powered nodes and they are required to work as long as possible (depending on the target application, a network lifetime from $24 \mathrm{~h}$ to several days is required). Thus, energy efficiency is a key requirement for such networks to maximise their lifetime. Routing protocols should select the best network path for data delivery. Since communication is one of the most energy consuming tasks in WSNs (Anastasi et al., 2009; Oberg and Xu, 2007), some nodes (of the most used paths) may quickly reach low levels of energy, which will turn them off and cause harm to the QoS provided by the network and ultimately compromising its lifetime. Since routing protocols obviously play an important role to guarantee energy efficiency and balance to the network, the routing algorithm must take into account several metrics and/or constraints on 
the process of path creation, the remaining energy of the node being one of them.

\subsection{Data heterogeneity and reporting}

In many situations, WSNs are assumed homogeneous regarding the data generated by each node. In their turn, BWSNs are highly heterogeneous. In patient monitoring applications, it is usual to have several nodes, each one generating different data types, depending on the specific application (e.g., heart rate, blood pressure and oximetry, or body temperature). In order to gather the data generated by the network it is necessary to define how they will be collected, i.e. the data report method. It depends on each application and also on the temporal requirements of the generated data. Data reporting can be classified as time-driven, event-driven, query-driven or a combination of these methods. Time-driven networks are used in applications that require periodic monitoring. An event-driven network reacts to unexpected and abrupt changes in sensed variables. Query-driven networks respond to queries generated by other nodes in the network. In realistic applications we have a hybrid of all these methods. The routing protocol should be able to deal with different data types and reporting methods.

\subsection{Bandwidth}

Bandwidth is a limited resource in all wireless applications, and even further in BWSNs. In the IEEE 802.15.4 technology (2.4 GHz), for instance, the bit rate is limited to $250 \mathrm{kbps}$. In typical applications of BWSNs, this limit can be easily reached; e.g., a BWSN for a 12 leads ECG monitoring of a single patient needs approximately $40 \mathrm{kbps}$ to transmit the raw samples (using a 16-bit ADC with a sampling rate of $250 \mathrm{~Hz}$ ). Despite such intensive network use, the routing protocol must contribute to the network performance, regardless of additional control and signalling traffic.

\subsection{Computational power and memory}

The limited memory and computational capabilities of WSN sensor nodes present new challenges to the design of routing protocols. These limitations will be reflected on the protocol complexity and performance thus, lightweight routing protocols with low computational requirements and low energy demands are required (Ehsan and Hamdaoui, 2011; Vasseur and Dunkels, 2010).

\section{Routing protocol for low-power and lossy networks}

The IETF ROLL working group has approved the new standard "IPv6 Routing Protocol for Low-power and Lossy Networks" in short, the RPL. The RPL introduces a series of novel mechanisms to allow efficient routing in resources constrained WSNs. Recent studies show that the RPL protocol performs well and may play an important role in the development of advanced monitoring applications designed for critical scenarios (Accettura et al., 2011; Ko et al., 2011a). Next, the key design principles on which RPL is built are explained, in particular how the RPL uses an objective function to specify the path generation to route data across the network. Since the proposed EAOF was designed to be used by the $\mathrm{RPL}$, this overview is essential to correctly understand the remaining of this paper.

The RPL is a Distance Vector routing protocol that specifies how to form a Direct Acyclic Graph (DAG) by using an OF and a set of constraints and metrics. Each DAG may be composed of one or more Destination Oriented Direct Acyclic Graph (DODAG), one per sink node. The DAG can be considered a logical topology over a physical network built to meet application specific criteria. RPL uses four identifiers to define and maintain a topology (Winter and Thubert, 2012): RPLInstanceID, which identifies each possible RPL instance running in the same WSN; DODAGID, which uniquely identifies one DODAG within one RPL instance; DODAGVersionNumber, which is incremented each time the DODAG is rebuilt; Rank, which reflects the distance from each node to the DODAG root.

To dynamically form and maintain a topology, the RPL uses a set of messages to disseminate information: the DODAG Information Solicitation (DIS), the DODAG Information Object (DIO), and the Destination Advertisement Object (DAO) (Winter and Thubert, 2012). These messages are broadcasted periodically by each node, and to control and minimise signalling overhead RPL uses the trickle algorithm (Levis et al., 2011), in each node, to trigger them.

DIO messages (Fig. 3) play an important role in RPL. They carry information about the DAG that allows the network nodes to discover a RPL instance and its configurations, such as RPLInstanceID, Version Number, Rank, OF and metrics. The Options field allows the DIO to carry several RPL Option Objects, two of which are the Metric Container (Fig. 4) and the DODAG Configuration (Fig. 5). The DODAG Configuration field OCP (Objective Code Point) identifies the OF used in the RPL instance. The Metric Container is used to disseminate metrics throughout the DODAG. A detailed description of the DIO messages format and its options can be found in Winter and Thubert (2012).

In order to build the RPL DAG, each node configured as DODAG root starts sending to its neighbours DIO messages with Rank

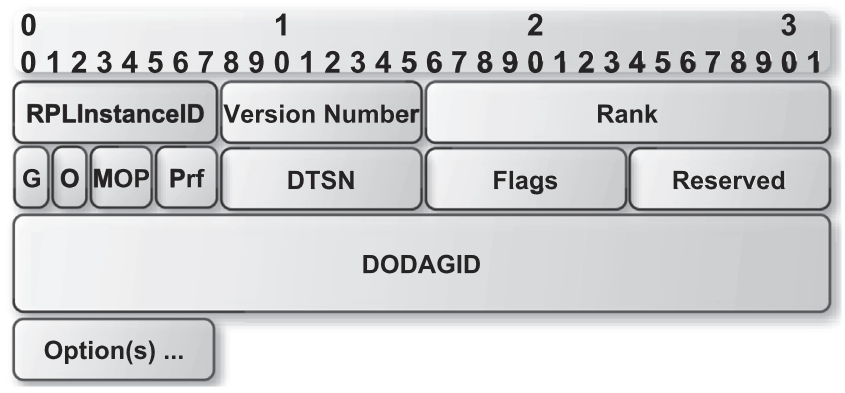

Fig. 3. The DIO Base Object used to disseminate through the network DAG-related information.

\begin{tabular}{|c|c|c|}
\hline Type $=2$ & Option Length & Metric Data ... \\
\hline
\end{tabular}

Fig. 4. The DAG Metric Container used to report metrics throughout the DODAG.

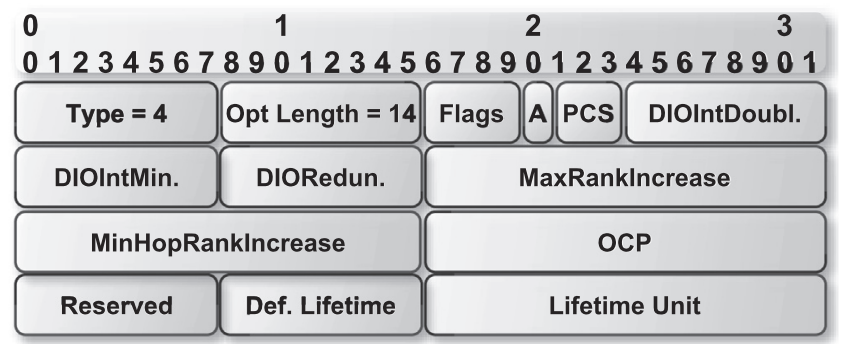

Fig. 5. The DODAG Configuration option used to disseminate configuration information throughout the DODAG. 
equal to one. Upon DIO reception, each node assesses whether the message should be processed or discarded. If the message is valid, the node updates its Rank and its cost to the root, and it updates its neighbours table and preferred parent. The node preferred parent must have a lower rank (to avoid loops) and it is selected according several possible OFs (Winter and Thubert, 2012).

As defined in Winter and Thubert (2012), the OF specifies how nodes within a RPL Instance select their parents based on routing metrics or constraints, and consequently they form paths to the DODAG root. Currently, there are two OFs specified as Internet Drafts by the ROLL working group, the Objective Function Zero (OF0) (Thubert, 2012) and the Minimum Rank Objective Function with Hysteresis (MRHOF) (Gnawali and Levis, 2012). The OF0 uses a hop count-based metric and the MRHOF uses hysteresis while selecting the path with the smallest metric value, e.g., the path ETX (De Couto et al., 2003).

There are two main implementations of the RPL protocol for real and simulated WSNs, the TinyRPL (Ko et al., 2011a) and the ContikiRPL (Tsiftes et al., 2010; Ko et al., 2011b). The ContikiRPL, which is used in this work, was developed for the Contiki OS. It includes all the fundamental mechanisms specified in the standard. However, it supports neither security features nor the dissemination of multiple metrics. Concerning the dissemination of multiple metrics, the necessary enhancements were made and the ContikiRPL is now able to support multiple metrics. The proposed EAOF, designed to be used by the RPL protocol, makes use of two metrics, the ETX and the remaining energy on each node.

\section{Proposed energy-aware objective function for RPL}

In this section, the design and implementation of the proposed EAOF is described. Let us consider a WSN with five sensor nodes and one sink (Fig. 6) where sensor nodes send periodically their messages to the sink. Since $S N_{3}, S N_{4}$ and $S N_{5}$ are the only ones in the sink radio range they have to forward the messages from $S N_{1}$ and $\mathrm{SN}_{2}$. Each node has three attributes: Rank, link ETX, and Remaining Energy (RE). Using the MRHOF and the ETX metric to build the route path to the sink, $S N_{1}$ and $S N_{2}$ will choose $S N_{4}$ as their best parent (it is the one with the lowest ETX). Consequently, $\mathrm{SN}_{4}$ will soon become inactive due to the energy depletion, and the network might not respond properly to subsequent messages. To avoid this, the proposed method takes into account not only the ETX metric but also the available energy on each node. The EAOF uses the link ETX and the RE on each node to compute the best path to the sink. This is the working principle: each node selects from its neighbours the nodes that have more reliable links (lowest ETX) to the sink. Then from that subset, the node having the maximum RE is selected to become the node's best parent. In this way, as will be demonstrated, the energy in the network will get balanced and the network lifetime will increase.

Figure 7 shows in detail the proposed EAOF algorithm. The parameters MAX_ETX and MIN_ENER are reconfigurable and dependent on the target application. The MAX_ETX represents the maximum ETX value that each neighbour can reach in order to be considered a best parent candidate. It is related with the Packets End-to-End Delay (E2ED), and depends on the application QoS requirements. The MIN_ENER is the minimum difference in the RE for a node to switch its best parent. It introduces some hysteresis in order to control how often the network will be reconfigured. It is used to improve the links stability thereby increasing the network performance. Finally, to avoid loops, each node rules out the neighbours with greater rank from being its best parent.

The EAOF configuration depends on the WSN application and its QoS requirements. Using the MAX_ETX and MIN_ENER parameters, the EAOF could be configurable to meet the specific requirements of each individual application.

Regarding the routing challenges and design issues faced when developing BWSNs, as presented in Section 3, the proposed EAOF was designed with them in mind. To mitigate the effect of the electromagnetic interferences faced by BWSNs when they are deployed in harsh environments as hospital facilities, the EAOF uses the ETX as metric to select the node within the most reliable path to the sink (i.e. the DODAG root) to be its best parent. In this way, the EAOF pursues the reduction of both the PER and the endto-end delay, depending on the retransmission policy in use. Furthermore, in cases wherein the nodes have some mobility, the routes are restored when necessary, while maintaining the criteria of choosing the most reliable paths to the sink. Unlike the existing implementations of the OF0 and the MRHOF, the EAOF uses the RE on each node to introduce energetic considerations when forming paths to route the data through the network. Being aware of the RE of each node, the routing protocol is able to promote the energy balance in the network while choosing the most reliable and most efficient paths. The use of these two metrics, the ETX and the RE made possible an increase in the network lifetime while maintaining high levels of QoS as required by BWSNs and their applications. When comparing the Contiki OS implementations of both the OF0 and the MRHOF with the EAOF, the computational requisites of such objective functions are similar since they perform the same algebraic operations. Nevertheless, when comparing the size of the code the EAOF needs more memory than the remaining ones (more $11 \%$ of program memory and $0.30 \%$ of data memory). Regarding the extra bandwidth required to broadcast the metrics across the network, only 2 bytes are added to the DAG Metric Container used to report metrics throughout the DODAG. In other words, both the additional traffic used and the computational complexity introduced by the EAOF are negligible.

\section{Experiment and evaluation}

Network simulators are extensively used to evaluate and compare the performance of routing protocols for WSNs (Liang, 2009). Their use has advantages and disadvantages. They allow easy and fast network deployments, controllable and flexible

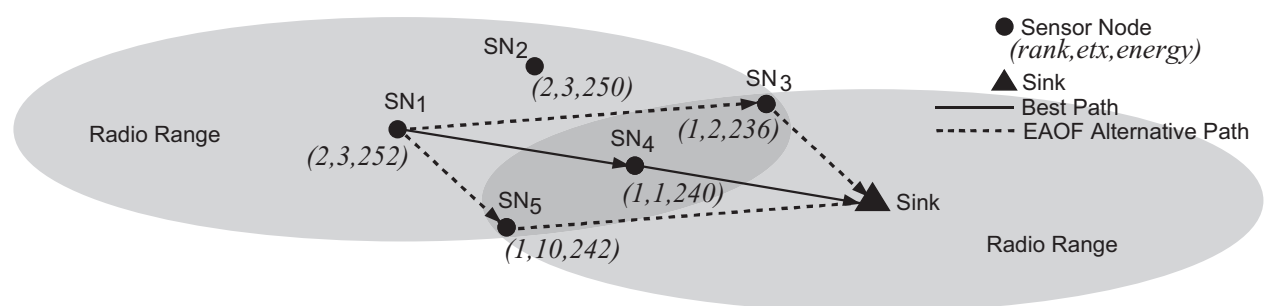

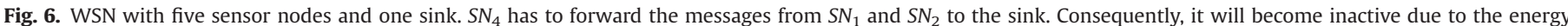
depletion. 


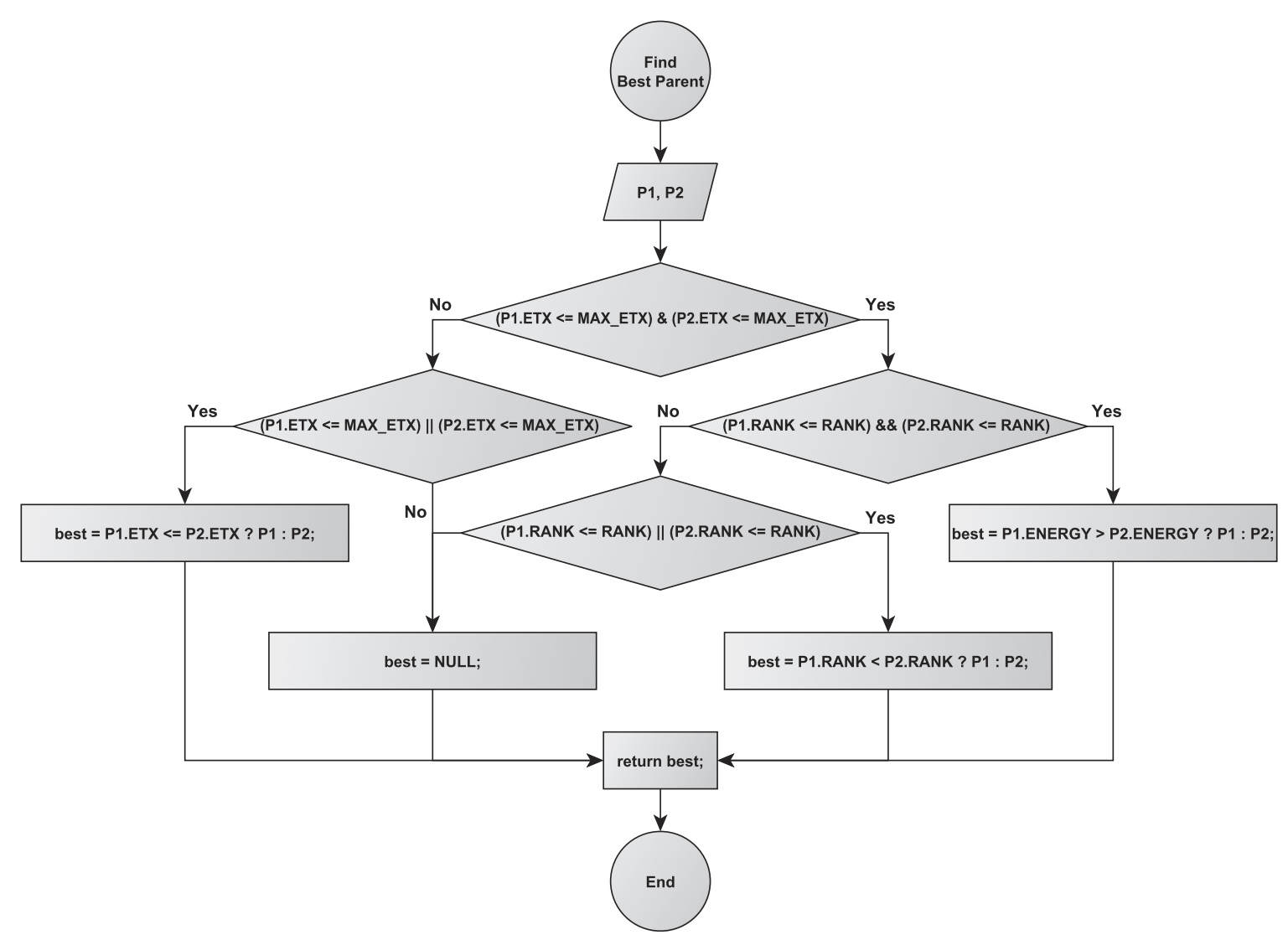

Fig. 7. To be the node best parent, the EAOF Algorithm selects the neighbour with the lowest ETX and the highest RE.

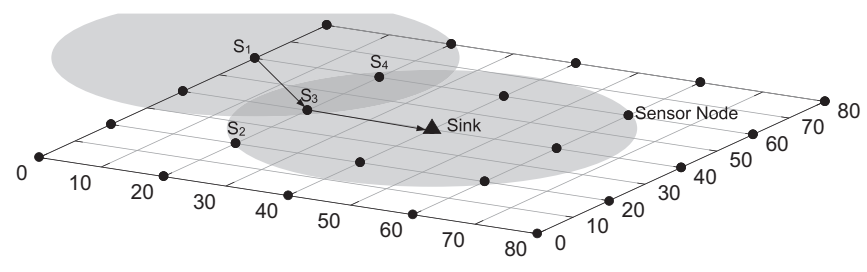

Fig. 8. Network deployment: sensor nodes are regularly distributed over an $80 \mathrm{~m} \times$ $80 \mathrm{~m}$ area. Each node has a radio range of $30 \mathrm{~m}$. The sink is at position $(40,40)$.

scenarios and the repeatability of the results obtained. However, due to the oversimplified channel models and protocols, simulations may not represent the reality; on the contrary, real deployments avoid problems relating to simplifications of models and protocols, but such deployments are considerably harder to implement and deploy. For these reasons, a hybrid approach based on the cross level emulation and simulation tool known as COOJA (Osterlind et al., 2006) has been used in this work. COOJA is a flexible WSNs simulator designed for simulating networks running Contiki OS (Dunkels et al., 2004).

To validate the EAOF, this section provides several simulation and the corresponding results. The comparison is with the ContikiRPL implementation of the MRHOF (Gnawali and Levis, 2012) using the ETX metric. The analysis consists of three parts. First, the Packet Reception Ratio (PRR) of both OFs is compared in order to verify their suitability for the target applications. Then, the energy efficiency of the network is analysed and conclusions are drawn concerning the energy imbalance in the network - in fact, it is possible to conclude that it decreases when using the EAOF. Finally, it is shown that the EAOF leads to a substantial growth of the network lifetime.
Table 1

Network configuration.

\begin{tabular}{ll}
\hline Network & \\
Deployment area & $80 \mathrm{~m} \times 80 \mathrm{~m}$ \\
Deployment type & $5 \times 5$ grid (see Fig. 7) \\
Number of nodes & 1 sink and 25 sensor nodes \\
Sink position & $(40,40) \mathrm{m}$ \\
Radio range & $30 \mathrm{~m}$ \\
Nodes initial energy & $10800 \mathrm{~mJ}$ \\
Discharge factor & 250 \\
Setup time & $60 \mathrm{~s}$ \\
Network layer & IPv6 with 6LowPAN \\
Transport layer & UDP \\
Routing protocol & RPL \\
Logical topology & Random \\
Application & \\
Task type & Time driven \\
Data length & $<70$ bytes (one packet) \\
Reporting interval (s) & $2,4,6,8,10,20,30,40,50,60$ \\
End to end delay & $\leq 80$ ms \\
Simulation & \\
Time & $900 \mathrm{~s}$ (PRR), 3600 s \\
Topologies & 10 \\
\hline
\end{tabular}

\subsection{Performance metrics}

In order to make a qualitative analysis and compare it with other solutions already available, the following figures of merit were defined:

Packet Reception Ratio: Due to the nature of their applications, BWSNs have to fulfil strong QoS requirements. Among such requirements, the application-level PRR is one of the most 
important. In this context, the EAOF has to achieve the same degree of performance when compared with the MRHOF.

Energy efficiency: In order to quantify and compare the energy balance and the efficiency of both objective functions, the time that each node spent transmitting or receiving data was measured. As stated in Dunkels et al. (2007), the energy consumption of a

Table 2

Simulation results and statistics.

\begin{tabular}{lccc}
\hline Metric & MRHOF & EAOF & Gain (\%) \\
\hline Packet reception ratio & $93.3 \%$ & $91.8 \%$ & -1.50 \\
Maximum energy consumption & $100 \%$ & $88.2 \%$ & 11.8 \\
Network lifetime (s) & 3850 & 4653 & 21 \\
\hline
\end{tabular}

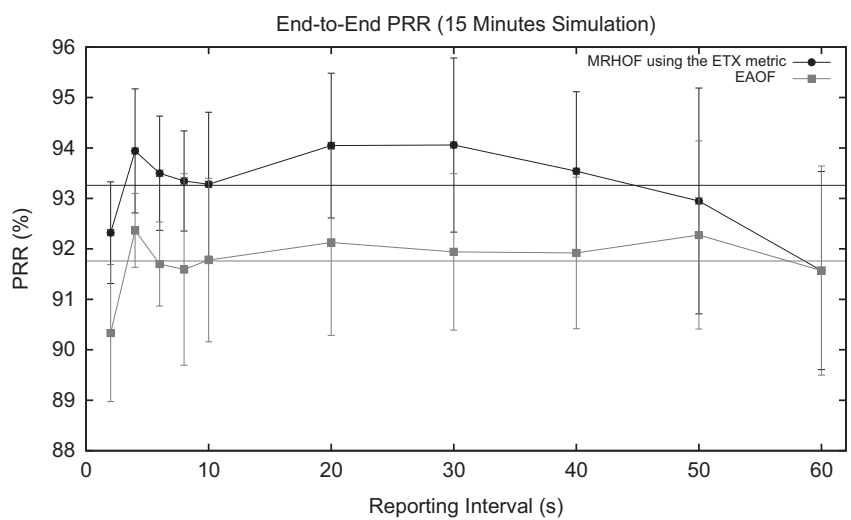

Fig. 9. Average and standard deviation of the packet reception ratio for different traffic loads (reporting intervals). node strongly depends on its radio activity but it can be estimated using a linear model, $E=\sum t_{i} p_{i}$, where $t_{i}$ and $p_{i}$ stand for the time and the power consumption of each component of the node. At the routing layer, the energy optimization is mainly done by acting on the radio activity. In this way, the time spent in communications may be used as an indirect measure of the energy consumed by each node, which will allow such time to be used in the study of the network energetic efficiency.

Network lifetime: Related as it is with network deployment, coverage, availability, connectivity and energy balance, the network lifetime of a WSN can be defined in many ways, as discussed in Dietrich and Dressler (2009). However, since BWSNs are made up of few nodes, they have low redundancy. This is why, in this work, the network lifetime has been defined as the time when the First Dead Node (FDN) appears.

\subsection{Simulation setup}

A case study in which a BWSN is used to perform a patient monitor and reporting task was tested in order to evaluate the EAOF. In such context, to maximise the covered area and, at the same time, minimise the probability of collisions and the effect of funnelling to the sink (border router), the BWSN was regularly deployed in a square area (Younis and Akkaya, 2008), as shown in Fig. 8. The scenario is a nursing room where 25 patients are being monitored and the data are sent to a local database. The 26 nodes (1 sink and 25 sensor nodes, one for each patient) have a radio range of $30 \mathrm{~m}$. After the network setup time, which is about $60 \mathrm{~s}$, each sensor node starts sending data packets at a specific rate. See Table 1 for a detailed description of the network and the simulation configurations. The BWSN was simulated using the framework presented in Abreu et al. (2012).
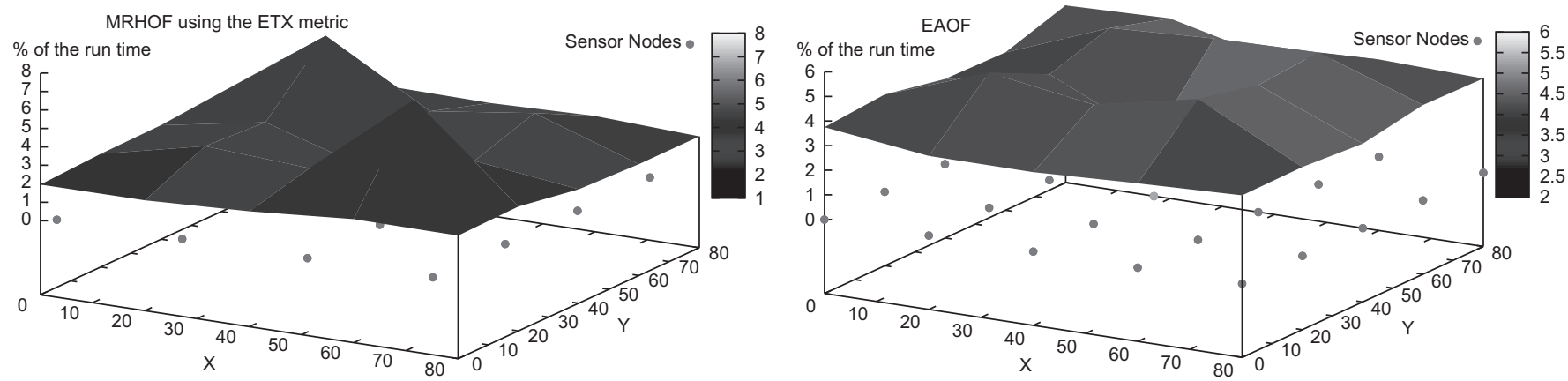

Fig. 10. Percentage of time that each node spends in communications (scenario in which each node transmits 0.5 packet/s).
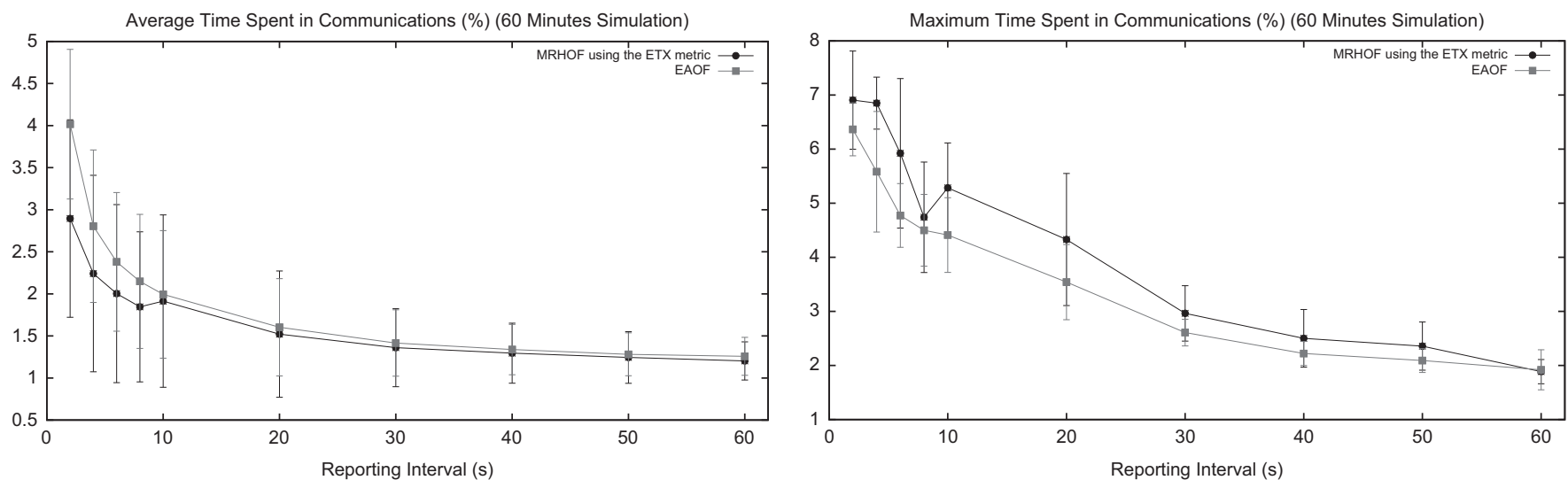

Fig. 11. Average and maximum time spent in communications in all scenarios. 
The case study was simulated for 10 different time-reporting scenarios where the reporting interval was configured to reflect different traffic scenarios when personal health parameters are been monitored using BWSNs. For each simulated scenario, 10 random routing topologies were created, thus obtaining 100 different DODAGs for each OF.

\subsection{Simulation results}

The results presented in Table 2 show significant improvements on the maximum energy consumption and the network lifetime, at the expense of a little degradation of the PRR. The Maximum Energy Consumption (MEC) parameter compares the energy consumed by the most active node within the network using the different OFs. Using the EAOF the most active node only spends $88.2 \%$ of the energy consumed by the equivalent node using the MRHOF. This saving increases the network lifetime by $21 \%$.

From the curves in Fig. 9, it is possible to conclude that the average PRR decrease is about $1.5 \%$. For each node, the MRHOF selects the best parent as the neighbour with the lowest ETX value so as to ensure that the number of retransmissions is minimised. On the other hand, the EAOF criterion is based on the best tradeoff between the ETX value and the remaining energy. Thus, the node's best parent will be the one with more remaining energy while presenting an acceptable ETX value. This is why the proposed strategy may lead to the use of longer or less reliable paths, which might result in a small degradation of the PRR. Depending on the application purpose and the QoS requirements, it may be necessary to improve the PRR; a possibility is to use a

Table 3

The EAOF increases the network lifetime by $21 \% . S_{n}$ stands for the simulation scenario in which each node transmits 1 packet every $n$ seconds.

\begin{tabular}{llll}
\hline \multicolumn{2}{l}{ First dead node time $(\mathrm{s})$} & & \\
\hline Reporting interval & MRHOF & EAOF & Gain (\%) \\
\hline$S_{2}$ & 2190 & 2470 & 13 \\
$S_{4}$ & 2490 & 3040 & 22 \\
$S_{6}$ & 2340 & 3300 & 41 \\
$S_{8}$ & 2700 & 3690 & 37 \\
$S_{10}$ & 2790 & 3541 & 27 \\
$S_{20}$ & 3710 & 4321 & 16 \\
$S_{30}$ & 4760 & 4961 & 4 \\
$S_{40}$ & 5030 & 6440 & 28 \\
$S_{50}$ & 7074 & 29 \\
$S_{60}$ & 5490 & 7692 & 10 \\
Average & 7002 & $\mathbf{4 6 5 3}$ & $\mathbf{2 1 \%}$ \\
\hline
\end{tabular}

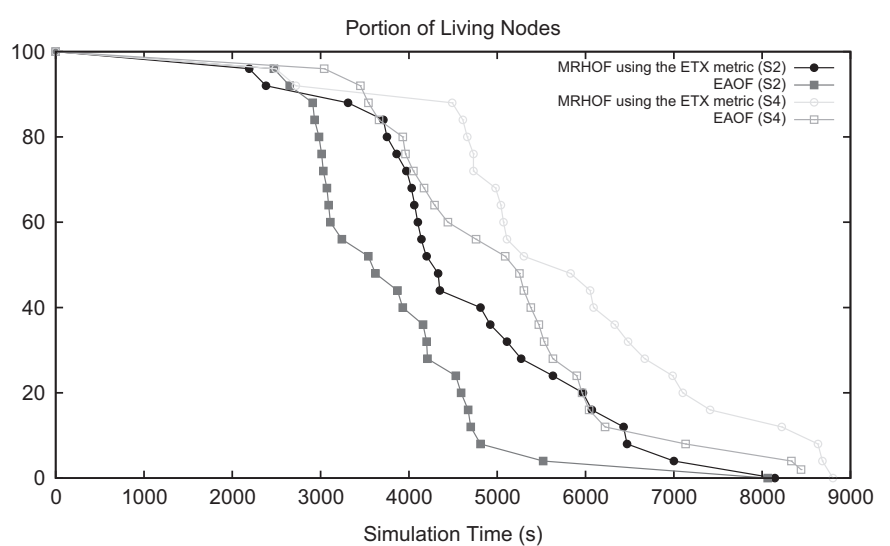

reliable transport protocol such as the Transmission Control Protocol (TCP). Since the results of both OFs are similar, the adopted strategy might be suitable for the two cases.

As stated before, the time that each sensor node spends in communications was used as an indirect measure of its energy consumption, balance, and efficiency. Figure 10 shows the percentage of time that each node spends transmitting or receiving data. Regarding the energy efficiency and balance, the EAOF presents better results than the MRHOF, which has high fluctuations in the energy consumption.

Figure 11 shows the average and maximum time spent in communications for each different reporting interval tested. It is possible to conclude that the average energy consumed by the network increases slightly. However, the peaks of energy consumption drop on an average of $11.8 \%$.

Regarding the network lifetime, several simulations were performed. The results display a significant improvement. Table 3 shows the network lifetime, for each simulated scenario, $S_{n}$.

Drawing upon the results presented, it is clear that EAOF provides a significant improvement to the network lifetime, with a minor impact on the PRR. This improvement allows longer periods of patient monitoring without human intervention. In addition, it will allow a better control of network QoS since node dying is avoided while maintaining the traffic distribution throughout the network (Fig. 12).

\section{Conclusion}

Although the emergent health technologies heavily rely on sensing devices attached to patients in order to monitor their health condition, there are still many technical problems to be resolved and also issues relating to users' acceptance and systems wearability. Such problems are mitigated when smaller devices are available. Since the energy module tends to use a significant volume of the overall sensing device, and considering that energy is one of the scarcest resources in WSNs, this work has focused on energy optimization with a view to the size reduction of the network sensor nodes. This paper has presented the design, implementation and evaluation of a new energy-aware objective function for the RPL routing protocol, the EAOF. The EAOF mitigates energy consumption and favours the energy balance in the network. Consequently, the network lifetime is increased allowing the use of smaller WSN nodes for patient monitoring purposes. The results obtained show that the proposed EAOF makes significant improvements in energy consumption, balance and network lifetime as compared to the ContikiRPL implementation of the MRHOF using the ETX metric. The improvement is

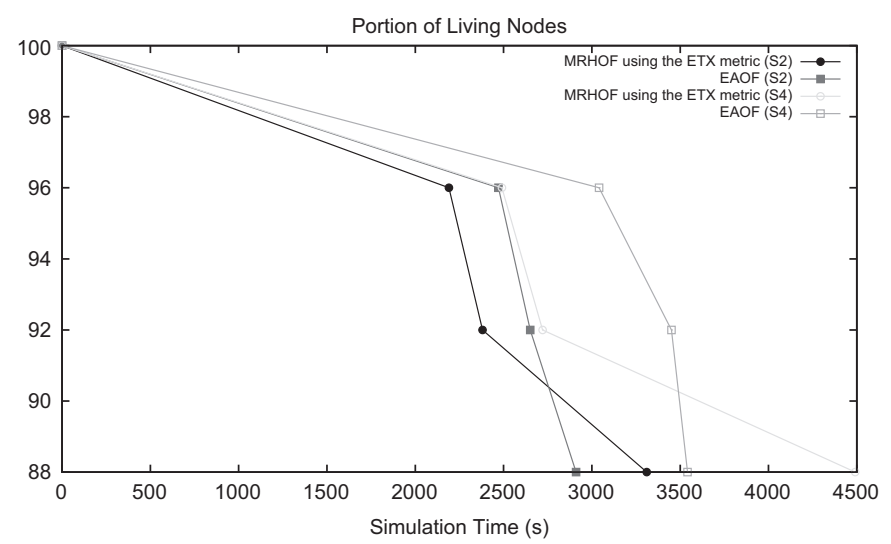

Fig. 12. Network lifetime for different scenarios. 
about $12 \%$ in the energy balance, and it is $21 \%$ in the network lifetime. It was also noticed that the EAOF leads to a slight degradation of $1.5 \%$ circa in the PRR, which could be mitigated by means of a strategy to improve the network PRR. In this way, the proposed solution would allow for a significant size reduction of the sensing device, thereby improving users' acceptance and wearability.

\section{Acknowledgements}

This work has been supported by the PhD grant of Portuguese Foundation for Science and Technology, FCT, SFRH/BD/61278/ 2009.

\section{References}

Abdulla AE, Nishiyama $\mathrm{H}$, Kato $\mathrm{N}$. Extending the lifetime of wireless sensor networks: a hybrid routing algorithm. Comput Commun 2012;35(9):1056-63 [Special Issue: Wireless Sensor and Robot Networks: Algorithms and Experiments].

Abreu C, Ricardo M, Mendes P. Framework for QoS performance assessment on biomedical wireless sensor networks. In: Biodevices 2012-international conference on biomedical electronics and devices. Vilamoura Portugal; 2012.

Accettura N, Grieco L, Boggia G, Camarda P. Performance analysis of the RPL routing protocol. In: 2011 IEEE international conference on mechatronics (ICM); 2011. p. 767-72.

Akyildiz I, Melodia T, Chowdury K. Wireless multimedia sensor networks a survey. Wireless Commun, IEEE 2007;14(6):32-9.

Anastasi G, Conti M, Francesco MD, Passarella A. Energy conservation in wireless sensor networks: a survey. Ad Hoc Networks 2009;7(3):537-68.

Bicakci K, Tavli B. Prolonging network lifetime with multi-domain cooperation strategies in wireless sensor networks. Ad Hoc Networks 2010;8(6):582-96.

De Couto DSJ, Aguayo D, Bicket J, Morris R. A high-throughput path metric for multi-hop wireless routing. In: Proceedings of the 9th annual international conference on mobile computing and networking. New York, NY, USA: ACM; MobiCom '03; 2003. p. 134-46.

Dietrich I, Dressler F. On the lifetime of wireless sensor networks. ACM Trans Sensors Netw 2009;5(1):5:1-39.

Dunkels A, Gronvall B, Voigt T. Contiki-a lightweight and flexible operating system for tiny networked sensors. In: 29th annual IEEE international conference on local computer networks; 2004. p. 455-62.

Dunkels A, Österlind F, Tsiftes N, He Z. Demo abstract: software-based sensor node energy estimation. In: Proceedings of the fifth ACM conference on networked embedded sensor systems (SenSys 2007). Sydney, Australia; 2007.

Ehsan S, Hamdaoui B. A survey on energy-efficient routing techniques with qos assurances for wireless multimedia sensor networks. IEEE Commun Surv Tutorials 2011(99):1-14 [Early Access].

Ferng HW, Tendean R, Kurniawan A. Energy-efficient routing protocol for wireless sensor networks with static clustering and dynamic structure. Wireless Pers Commun 2012;1:1-2, http://dx.doi.org/10.1007/s11277-011-0260-4.

Gnawali O, Levis P. The minimum rank objective function with hysteresis, RFC 6719; 2012.

Hadjidj A, Souil M, Bouabdallah A, Challal Y, Owen H. Wireless sensor networks for rehabilitation applications: challenges and opportunities. J Netw Comput Appl 2013;36(1):1-15.

Hui J, Thubert P. Compression format for IPv6 datagrams over IEEE 802.15.4-based networks. RFC 6282 (Proposed Standard); 2011.
Ko J, Dawson-Haggerty S, Gnawali O, Culler D, Terzis A. Evaluating the performance of RPL and 6LoWPAN in TinyOS. In: Proceedings of the workshop on extending the internet to low power and lossy networks. IPSN'11; 2011a.

Ko J, Eriksson J, Tsiftes N, Dawson-Haggerty S, Terzis A, Dunkels A, Culler D. Contikirpl and tinyrpl: happy together. In: Proceedings of the workshop on extending the internet to low power and lossy networks (IPSN 2011). Chicago, IL, USA; 2011b.

Ko J, Terzis A, Dawson-Haggerty S, Culler D, Hui J, Levis P. Connecting low-power and lossy networks to the internet. Commun Mag IEEE 2011c;49(4):96-101.

Lai CC, Lee RG, Hsiao CC, Liu HS, Chen CC. A h-qos-demand personalized home physiological monitoring system over a wireless multi-hop relay network for mobile home healthcare applications. J Netw Comput Appl 2009;32 (6):1229-41.

Levis P, Clausen T, Hui J, Gnawali O, Ko J. The trickle algorithm. RFC 6206 (Proposed Standard); 2011.

Liang X. QoS provisioning for wireless sensor networks: algorithms, protocols and modeling [Ph.D. thesis]. Interventional Center, University of Oslo; 2009.

Lindsey S, Raghavendra C. Pegasis: power-efficient gathering in sensor information systems. In: Aerospace conference proceedings, IEEE, vol. 3; 2002. p. 3-1125-30

Liu T, Li Q Liang P. An energy-balancing clustering approach for gradient-based routing in wireless sensor networks. Comput Commun 2012;35(17):2150-61, http://dx.doi.org/10.1016/j.comcom.2012.06.013.

Oberg L, Xu Y. A complete energy dissipation model for wireless sensor networks. In: Proceedings of the international conference on sensor technologies and applications sensorComm; 2007. p. 531-40.

Ok C, Lee S, Mitra P, Kumara S. Distributed routing in wireless sensor networks using energy welfare metric. Inf Sci 2010;180(9):1656-70.

Ok CS, Lee S, Mitra P, Kumara S. Distributed energy balanced routing for wireless sensor networks. Comput Ind Eng 2009;57(1):125-35 [Collaborative e-Work Networks in Industrial Engineering].

Osterlind F, Dunkels A, Eriksson J, Finne N, Voigt T, Cross-level sensor network simulation with cooja. In: Proceedings 2006 31st IEEE conference on local computer networks; 2006. p. 641-8.

Preve N. Ubiquitous healthcare computing with sensor grid enhancement with data management system (SEGEDMA). J Med Syst 2011;35:1375-92.

Ren F, Zhang J, He T, Lin C, Ren S. EBRP: energy-balanced routing protocol for data gathering in wireless sensor networks. IEEE Trans Parallel Distributed Syst 2011;22(12):2108-25.

Shah R, Rabaey J. Energy aware routing for low energy ad hoc sensor networks. In: Wireless communications and networking conference, 2002. WCNC2002, vol. 1. IEEE; 2002. p. 350-5.

Tao M, Lu D, Yang J. An adaptive energy-aware multi-path routing protocol with load balance for wireless sensor networks. Wireless Pers Commun 2012;63: 823-846, http://dx.doi.org/10.1007/s11277-010-0169-3.

Thubert P. RPL objective function zero, RFC 6552; 2012.

Tsiftes N, Eriksson J, Dunkels A. Poster abstract: low-power wireless IPv6 routing with ContikiRPL. 〈http://soda.swedish-ict.se/3849/>; 2010.

Vasseur J, Kim M. Routing metrics used for path calculation in low power and lossy networks, RFC 6551; 2012.

Vasseur JP, Dunkels A. Interconnecting smart objects with IP-the next internet Morgan Kaufmann; 2010.

Watteyne T, Molinaro A, Richichi MG, Dohler M. From manet to IETF rol standardization: a paradigm shift in WSN routing protocols. IEEE Commun Surv Tutorials 2011;13(4):688-707.

Winter T, Thubert P. Rpl: Ipv6 routing protocol for low power and lossy networks, RFC 6550; 2012.

Xu Y, Heidemann J, Estrin D. Geography-informed energy conservation for ad hoc routing. In: Proceedings of the 7th annual international conference on mobile computing and networking. New York, NY, USA: ACM; MobiCom '01; 2001 p. $70-84$.

Younis M, Akkaya K. Strategies and techniques for node placement in wireless sensor networks: a survey. Ad Hoc Networks 2008;6(4):621-55. 